ARTigo Original

Original Article

\title{
Baixa Incidência de Fraturas do Quadril Associadas à Osteoporose, em Sobral-CE ${ }^{(*)}$
}

\section{Low Incidence of Hip Fractures Associated with Osteoporosis in Sobral-CE}

\author{
Francisco Airton Castro da Rocha ${ }^{(1)}$ e Ana Regina Ribeiro ${ }^{(2)}$
}

\section{RESUMO}

Objetivo: fraturas do quadril em pacientes acima de 60 anos representam séria morbidade ligada à osteoporose. Influências genéticas e ambientais foram relatadas como associadas à epidemiologia da osteoporose. Sobral, localizada no nordeste do Brasil ( $\left.3^{\circ} 41^{\prime} 10^{\prime \prime} \mathrm{S} / 40^{\circ} 20^{\prime} 59^{\prime \prime} \mathrm{E}\right)$, tem 138.565 mil habitantes que são predominantemente descendentes de brancos portugueses e índios brasileiros. Métodos: avaliamos a ocorrência de fraturas do quadril em Sobral entre julho de 1996 e junho de 2000. Os dados foram obtidos dos prontuários médicos da Santa Casa de Sobral, que é o hospital de referência local. Todos os registros de pacientes maiores de 20 anos com diagnóstico de fratura do fêmur ou do quadril foram revisados. Resultados: identificamos no total 79 fraturas. Dez (12,6\%), que ocorreram em menores de 50 anos, foram excluídas da análise. Sessenta e nove $(87,3 \%)$ ocorreram em maiores de 50 anos, compreendendo 19 (27,5\%) homens e $50(72,4 \%)$ mulheres, com uma incidência anual idadeajustada de 5,59/10.000/ano em homens e 12,4/10.000/ano em mulheres, respectivamente, para essa faixa etária. Dois pacientes, de 79 e 82 anos, morreram 9 e 6 dias após hospitalização, respectivamente. A média de tempo de hospitalização foi de 7,5 dias (variação de 4-19 dias). Conclusões: Houve tendência a acréscimo na incidência de fraturas de quadril em pessoas acima de 50 anos. A incidência de fraturas do quadril nessa população foi cerca de quatro vezes menor que a relatada em brancos caucasóides.

Palavras-chave: osteoporose, fraturas, quadril, epidemiologia.

\begin{abstract}
Objective: Hip fractures in patients older than 60 years old represent a serious morbidity linked to osteoporosis. Both genetic and environmental influences have been reported as important factors related to the epidemiology of osteoporosis. Sobral is located in the northeast of Brazil ( $3^{\circ} 41^{\prime} 10^{\prime \prime} S / 40^{\circ} 20^{\prime} 59^{\prime \prime} E$ ) and has 138,565 inhabitants, which are mostly descendants from white Portuguese and native Brazilian. Methods: We evaluated the occurrence of hip fractures in Sobral between July/1996 and June/2000. This was a retrospective cohort based study. Data were obtained from the medical records of the Santa Casa de Sobral that is the reference hospital in this area. All chart records of patients aged more than 20 years old with a diagnosis of femoral or hip fracture were revised. Results: A total of 79 fractures were identified. Ten (12.6\%) cases that occurred in patients less than 50 years old were excluded from further analysis. Sixty-nine cases (87.3\%) occurred in patients aged more than 50 years old comprising 19 (27.5\%) men and 50 (72.4\%) women giving an age-adjusted annual incidence rate of 5.59/10,000/ year in men and 12.4/10,000/year in women, respectively for people in this age range. Two patients, aged 79 and 82, died 9 and 6 days after hospital admission, respectively. The average time of hospitalization was 7.5 days (range 4-19 days). Conclusions: There was a tendency to a rise in the incidence of hip fractures in people older than 50 years old. Interestingly, the hip fracture rate in this population is considerably lower (around four times less) than the reported rates in white Caucasians.
\end{abstract}

Keywords: osteoporosis, fracture, hip, epidemiology.

\footnotetext{
* Disciplina de Reumatologia da Faculdade de Medicina da Universidade Federal do Ceará (FMUFC). Este trabalho contou com o apoio do Fundo de Auxílio à Pesquisa e Ensino em Reumatologia da Sociedade Brasileira de Reumatologia. Recebido em 09/01/2003. Aprovado, após revisão, em 15/03/2003.

1. Disciplina de Reumatologia da FMUFC.

2. Disciplina de Reumatologia da FMUFC.
}

Endereço para correspondência: Dr. Francisco Airton Castro da Rocha. Rua Tiburcio Cavalcante, 2100/1201, CEP 60125-101,Fortaleza, Ceará, Brasil. E-mail: arocha@ufc.br 


\section{INTRODUÇÃO}

A osteoporose é a mais freqüente entre as doenças osteometabólicas. Os níveis de incidência de fraturas do quadril, a mais grave morbidade relacionada à osteoporose, variam consideravelmente entre países, sendo muito mais freqüentes em brancos caucasóides. Nos países em desenvolvimento, os dados são escassos e o impacto socioeconômico da osteoporose ainda não está bem definido. Dados da literatura revelam grande variação da incidência de fraturas do quadril em diferentes países. As influências genéticas e ambientais estão ligadas a essa variabilidade ${ }^{(1-5)}$. A identificação de populações de baixo risco para osteoporose pode ajudar a identificar fatores genéticos ou ambientais que possam estar associados com um "osso melhor".

No Brasil, foi realizado um estudo relatando que a incidência idade-ajustada em Marília (SP) foi de 28,8 e 12,6 fraturas de quadril/10.000 habitantes/ano para mulheres e homens acima de 60 anos, respectivamente ${ }^{(6)}$. Em virtude das dimensões continentais do Brasil e da heterogeneidade da formação étnica, é relevante estudar possíveis variações na incidência de fraturas associadas à osteoporose em nosso país.

A cidade de Sobral - CE (341'10” S/ 40²0’59” E) está localizada no Nordeste do Brasil e possui uma população de 138.565 mil habitantes, segundo o último censo do Instituto Brasileiro de Geografia e Estatística (IBGE). A maioria da população é formada por descendentes de brancos portugueses e nativos brasileiros. A média de temperatura anual é de $26,6^{\circ} \mathrm{C}$ e a média de chuvas é de 821,6 $\mathrm{mm} /$ ano. A cidade tem um serviço bem montado de saúde pública e há apenas um grande hospital para onde são encaminhados todos os casos de fraturas.

Realizamos um estudo em Sobral-CE, de 1996 a 2000, para investigar a incidência de fraturas de quadril nessa população.

\section{PACIENTES E MÉTODOS}

Estudo de uma coorte retrospectiva. Todos os pacientes admitidos na Santa Casa de Misericórdia de Sobral com diagnóstico de fratura do fêmur proximal foram inicialmente incluídos. Todos os casos foram considerados como sendo fraturas de quadril. Todos os endereços dos pacientes foram verificados para garantir que apenas os residentes em Sobral fossem incluídos.

Os objetivos primários do estudo foram determinar os seguintes índices de incidência para fraturas de quadril: taxa bruta para incidência na população acima de 20 anos de idade; incidência idade-ajustada para pessoas entre 20 e 49 anos, 50 e 59 anos e maiores de 60 anos de idade; incidência idade-ajustada para pessoas maiores de 60 anos de idade.

Os critérios de exclusão foram: fraturas resultantes de trauma grave em acidente automobilístico, fraturas patológicas por doenças metastáticas ou tumores primários ósseos. Todos os casos tiveram seus prontuários revisados na admissão e na alta hospitalar para verificação do diagnóstico final.

\section{RESULTADOS}

Um total de 79 fraturas foram identificadas. Dez fraturas $(12,7 \%)$ ocorreram em indivíduos menores de 50 anos de idade. Vinte e nove fraturas $(36,7 \%)$ ocorreram em homens e cinquenta $(63,3 \%)$ ocorreram em mulheres, resultando numa proporção de 1:1,7 na relação homem/mulher.

A incidência idade-específica das fraturas de quadril aumentou rapidamente após os 50 anos de idade, para ambos os sexos, embora o aumento tenha sido mais relevante nas mulheres acima de 60 anos (Tabelas 1 e 2 e Figura 1).

A Tabela 3 compara a incidência idade-ajustada obtida no presente estudo com a observada em outros estudos semelhantes no mundo, mostrando uma incidência muito baixa para os dois estudos conduzidos no Brasil, incluindo o realizado em Marília (SP) e o presente estudo em Sobral (CE).

A maioria dos pacientes recebeu tratamento cirúrgico (74 dos 79), compreendendo 93,75\% dos casos. A média de internação hospitalar foi de 7,2 dias e houve dois óbitos relacionados à ocorrência da fratura (2,5\% do total de casos). Um desses pacientes era um homem de 79 anos de idade e outro era uma mulher de 82 anos, que faleceram 9 e 6 dias após a hospitalização, respectivamente.

TABELA 1

INCIDÊNCIA MÉDIA ANUAL DE FRATURAS DO QUADRIL EM HOMENS, EM SOBRAL (DE JULHO DE 1996 A JUNHO DE 2000)

\begin{tabular}{lccc}
\hline $\begin{array}{l}\text { Grupo etário } \\
\text { (anos) }\end{array}$ & Total de casos & População & $\begin{array}{c}\text { Incidência anual } \\
\text { (por 10.000) }\end{array}$ \\
\hline $20-49$ & 6 & 25.123 & 0.6 \\
\hline $50-59$ & 2 & 3.699 & 1.4 \\
$60-69$ & 5 & 2.649 & 4.7 \\
$70-79$ & 6 & 1.483 & 10.1 \\
$>80$ & 6 & 660 & 22.7 \\
\hline
\end{tabular}


TABELA 2

INCIDÊNCIA MÉDIA ANUAL DE FRATURAS DO QUADRIL EM MULHERES, EM SOBRAL (DE JULHO DE 1996 A JUNHO DE 2000)

\begin{tabular}{lccc}
\hline $\begin{array}{l}\text { Grupo etário } \\
\text { (anos) }\end{array}$ & Total de casos & População & $\begin{array}{c}\text { Incidência anual } \\
\text { (por 10.000) }\end{array}$ \\
\hline $20-49$ & 4 & 28.550 & 0.4 \\
\hline $50-59$ & 3 & 4.400 & 1.7 \\
\hline $60-69$ & 9 & 3.054 & 7.4 \\
\hline $70-79$ & 17 & 1.773 & 24 \\
\hline 80 & 21 & 846 & 62 \\
\hline
\end{tabular}

TABELA 3

COMPARAÇÃO DA INCIDÊNCIA DE FRATURAS DE QUADRIL EM Sobral - CE, Brasil (de JUlho de 1996 a junho de 2000) COM A INCIDÊNCIA EM OUTRAS POPULAÇÕES DO MUNDO

\begin{tabular}{lcc}
\hline População (ano) & Mulheres & Homens \\
\hline Oslo (NOR - 1978-79) & 122 & 50,1 \\
\hline Rochester (USA - 1965-74) & 81,7 & 29,7 \\
Columbia (USA - 1980) & 65,4 & 15,7 \\
\hline Reino Unido (UK -1974) & 63,2 & 18,4 \\
\hline Picardy (FRA - 1987) & 44,4 & 18 \\
\hline Marília (SP/BRA - 1995) & 28,8 & 12,6 \\
\hline Sobral (CE/BRA - 1996-00)* & 20,7 & 8,9 \\
\hline Siena (ITA - 1975-85) & 3 & 0,7
\end{tabular}

Incidência idade-ajustada por ano/10.000 habitantes. (Maiores de 60 anos) (Modificado de Komatsu, et al, 1995).

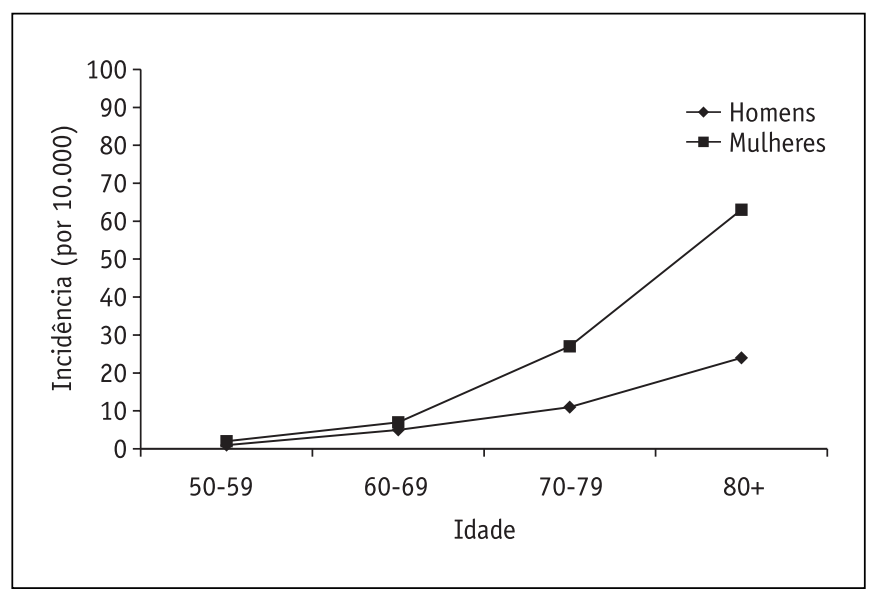

FIGURA 1 - Incidência de fraturas de quadril entre homens e mulheres em Sobral (CE), Brasil, de julho de 1996 a junho de 2000.

\section{DISCUSSÃO}

A grande variabilidade na incidência de fraturas relacionadas à osteoporose tem sido associada a influências ambientais e genéticas. Fatores raciais, geográficos e invernos rigorosos, bem como hábitos alimentares, atividades físicas e comorbidades que levam ao uso de sedativos parecem influenciar a incidência de fraturas no idoso ${ }^{(1,7,9)}$.

Dados da literatura mostrando que fatores genéticos influenciam a densidade mineral óssea ${ }^{(8,9)}$ sugerem sua importância na patogênese da osteoporose. Esses dados indicam que a identificação de populações de baixo risco para desenvolver fraturas de quadril possa ajudar no estabelecimento de fatores protetores para melhorar a prevenção e o tratamento da osteoporose. Em estudo recente, demonstrou-se que indivíduos de herança africana têm maior variabilidade na densidade mineral óssea do que poderia ser explicado, mais por fatores ligados à raça que a variações ambientais ${ }^{(8)}$. Em outro estudo recente, a incidência de fraturas de membros variou substancialmente em diferentes países europeus ${ }^{(5)}$

O presente estudo é a primeira descrição de uma baixa incidência de fraturas de quadril em uma população vivendo em uma região equatorial. Comparando-se com o estudo realizado em Marília (SP), observamos que, apesar de tão distantes entre si, cerca de 3.500 quilômetros, com climas bem diferentes, Marília e Sobral apresentaram uma incidência de fraturas do quadril significativamente menor que a observada em estudos similares em outras cidades no mundo ${ }^{(6)}$.

É pouco provável que a menor expectativa de vida no Brasil justifique essa baixa incidência, pois os dados foram ajustados para as diferentes faixas etárias, e em todas elas o risco foi menor que o observado em outros países. Este estudo nos motivou a iniciar um projeto mais amplo a fim de investigar possíveis fatores genéticos ligados aos índios brasileiros que possam influenciar essa baixa incidência de fraturas de quadril relacionadas à osteoporose na nossa população.

\section{AGRADECIMENTOS}

Os autores agradecem à Secretaria de Saúde da Prefeitura Municipal de Sobral e à diretoria da Santa Casa de Misericórdia de Sobral o apoio ao projeto e o acesso irrestrito às suas informações. Os autores também agradecem ao dr. Luis Eduardo C. Andrade (Unifesp/EPM) a revisão crítica do manuscrito. 


\section{REFERÊNCIAS}

1. Baudoin C, Fardellone, P, Potard V, et al: Fractures of the proximal femur in Picardy, France, in 1987. Osteoporosis Int 3: 43-9, 1993.

2. Boyce WJ, Vessey MP: Rising incidence of fracture of the proximal femur. Lancet 19: 150-1, 1985.

3. Cooper C, Campion G, Melton LJIII: Hip fractures in the elderly: a worldwide projection. Osteoporosis Int 2: 285-9, 1992.

4. Gennari C, et al: Trends in the incidence of hip fracture in Siena, Italy, from 1980-1991. Bone 14: 31-4, 1993.

5. Ismail AA, Pye SR, Cockerill WC, et al: Incidence of limb fracture across Europe: Results from the European prospective osteoporosis study (EPOS). Osteoporosis Int 13: 565-71, 2002.
6. Komatsu RS, Simões MFJ, Ramos LR, Szejnfeld VL: Incidence of fractures of the proximal femur in Marilia, São Paulo, Brazil, 1994 and 1995. Rev Bras Reumatol 39: 325-31, 1999.

7. Ling X, Aimin L, Xihe Z, et al: Very low rates of hip fractures in Beijing, People's Republic of China. Am J Epidemiol 144: 901-7, 1996.

8. Melton LJ, Marquez MA, Achebach SJ, et al: Variations in bone density among persons of African heritage. Osteoporosis Int 13: 55159, 2002.

9. Nguyen TV, Blangero J, Eisman JA: Genetic epidemiological approaches to the search for osteoporosis genes. J Bone Miner Res 15: 392-401, 2000. 